\title{
A Rare Case Presentation on Langerhan's Cell Histiocytosis an Chronic Disseminated Form with Gingival Enlargement in Three and a Half Year Old Pediatric Patient
}

\section{Manjul Tiwari*}

School of Dental Sciences, Sharda University, Uttar Pradesh, India

*Corresponding author: Manjul Tiwari, Assistant professor, Department of Oral Pathology and Microbiology, School of Dental Sciences, Sharda University, Uttar Pradesh, India, E-mail: manjultiw@gmail.com

Rec date: Jan 04, 2016; Acc date: Feb 28, 2016; Pub date: Mar 05, 2016

Copyright: (C) 2016 Tiwari M. This is an open-access article distributed under the terms of the Creative Commons Attribution License, which permits unrestricted use, distribution, and reproduction in any medium, provided the original author and source are credited.

Abstract
Langerhan's Cell Histiocytosis formerly known as histiocytosis X traditionally denotes a group of diseases that
stem from proliferative reticuloendothelial disturbances. The etiology and pathogenesis of the disease remain
debatable.The present paper reports a case occuring in a three and a half year old ( $31 / 2$ year pediatric) child
reported to the department of oral pathology with gingival enlargement of the jaws, also disscussed are the
radiological features along with histopathological features of the case.

Keywords: Histocytosis X; Langerhan's cell; Histocytosis; Exopthalomos; Diabetes insipidus

\section{Introduction}

Langerhan's cell histiocytosis (LCH), formerly known as Histiocytosis $\mathrm{X}$ is currently considered as disorder of immune regulation manifested by abnormal proliferation of histiocytosis and granuloma formation.

The Langerhan's cell, a unique histiocyte is the distinctive pathologic component of the disease. LCH may affect any organ, although the reticuloendothelial system (i.e. bones, skin, lymph nodes, liver and spleen) is involved in most cases [1].

Langerhan's cell disease manifests in three forms:

Acute disseminated form: It is previously referred to Letterer-siwedisease most likely represents a malignant neoplastic process. It is characterized by a rapidly progressive, clinical course and widespread in organs, bones and skin involvement by the proliferative process in infants has been the common presentation.

Chronic disseminated form: It is previously referred to as HandSchullers-Christian syndrome with the classical triad of lytic bone lesions, exophthalmos and diabetes insipidus.

Chronic Localized form: With only unifocal or multifocal bone lesions previously termed as eosinophilic granuloma [2].

Etiopathogenesis: The pathogenesis of $\mathrm{LCH}$ is unknown. It may be caused due to hypersensitive reaction to unknown antigen with stimulation of histiocytes-macrophage system.

Deficiency of suppressor lymphocytes (T-8, altered immunoglobulin's autoantibodies and structural changes to thymus in all the advanced forms have been found in $\mathrm{LCH}$ patients.

An inflammatory origin is also suspected due to the microscopic characteristics and clinical evolution, a bacterial origin although no specific causal microorganism has been identified [3].
Here I report a case of chronic disseminated form of Langerhan's cell disease (Hand-Schullers-Christian disease) presenting with gingival enlargement in $3 \frac{1}{2}$ year old pediatric patient.

\section{Case History}

A three and half year old male child was referred to department of oral pathology with swelling, pain and mobility involving deciduous canine and first molar region involving all the four quadrants since five months. The patient's father further gave the history of ulcers and inability to tolerate hot food. There was no history of trauma or any associated family history.

General examination of the patient with paediatrician and ENT surgeon revealed presence of Hepatosplenomegaly and Otitis media (Figure 1).

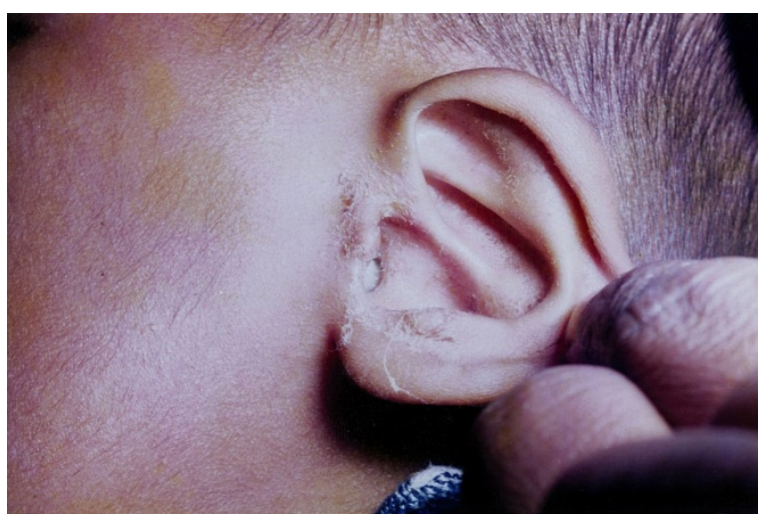

Figure 1: Preauricular pus discharge suggestive of Otitis Media.

Extra oral examination revealed right submandibular lymphadenopathy however there was no exopthalmos. Intraorally gingival enlargement was seen in molar region in all the four quadrants. Each enlargement was around $1.52 \mathrm{~cm}$ in size, diffuse 
Citation: Tiwari M (2016) A Rare Case Presentation on Langerhan's Cell Histiocytosis an Chronic Disseminated Form with Gingival Enlargement in Three and a Half Year Old Pediatric Patient. Oral health case Rep 2: 108. doi:10.4172/OHCR.1000108

Page 2 of 3

reddish in colour and soft to firm in consistency, bleeding was evident on slightest provocation (Figure 2).

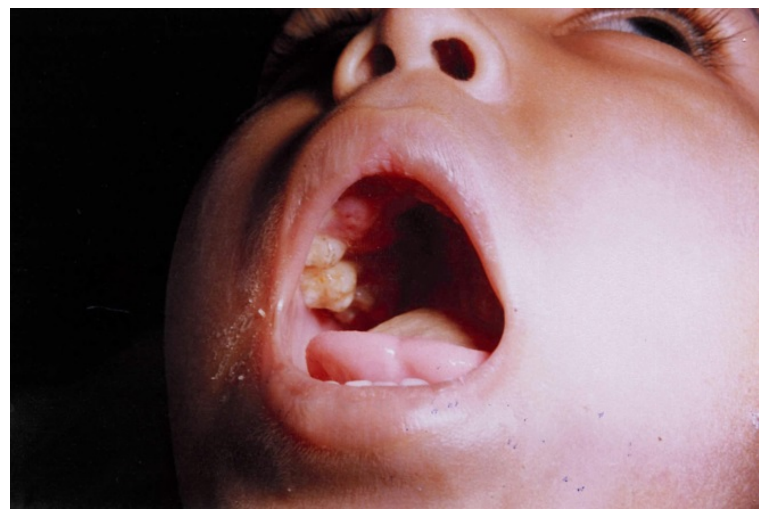

Figure 2: Intra oral pictures showing gingival enlargement.

Radiographic examination showed punched out lytic lesions in the skull involving parietal, temporal and frontal bones. In jaws there was diffuse destruction of bone and displacement of teeth (Figure 3).

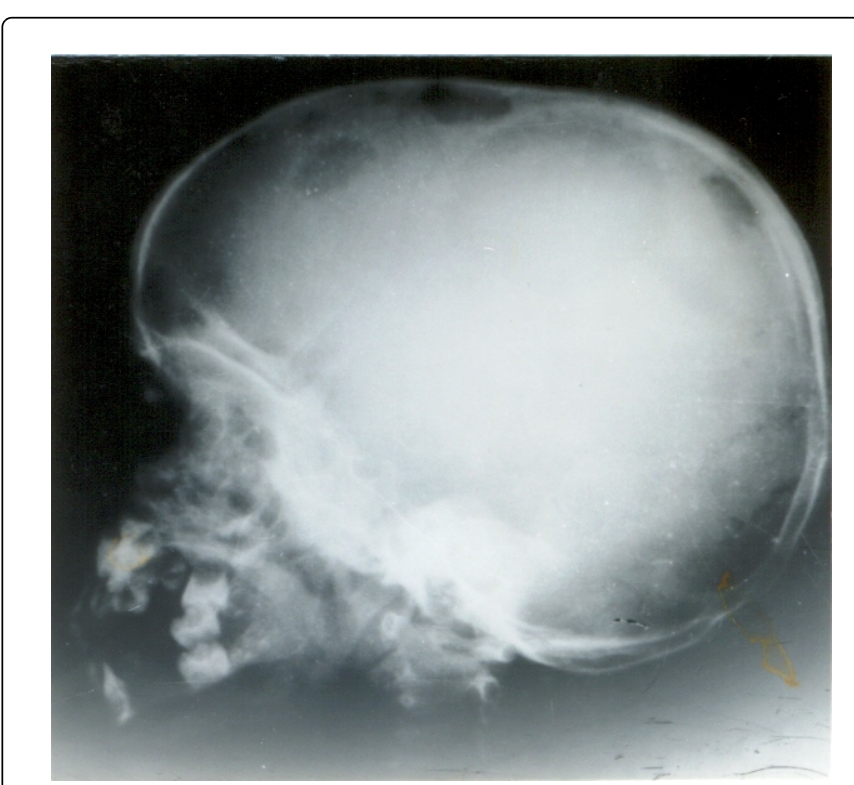

Figure 3: Radiograph (Lateral view) shows multiple punched out lesions in skull.

Lab investigations were within normal limits which included CBC, LFT, coagulation studies and urine osmolality.

Microscopic examination of the lesion revealed presence of many histiocyte like cells, eosinophils and lymphocytes, diffusely scattered throughout the connective tissue stroma, keeping the clinical, radiological and histopathological features in mind a diagnosis of Langerhan's cell disease (Histiocytosis X) in general and Handschullers-Christian disease in particular was made(Figures 4 and 5).

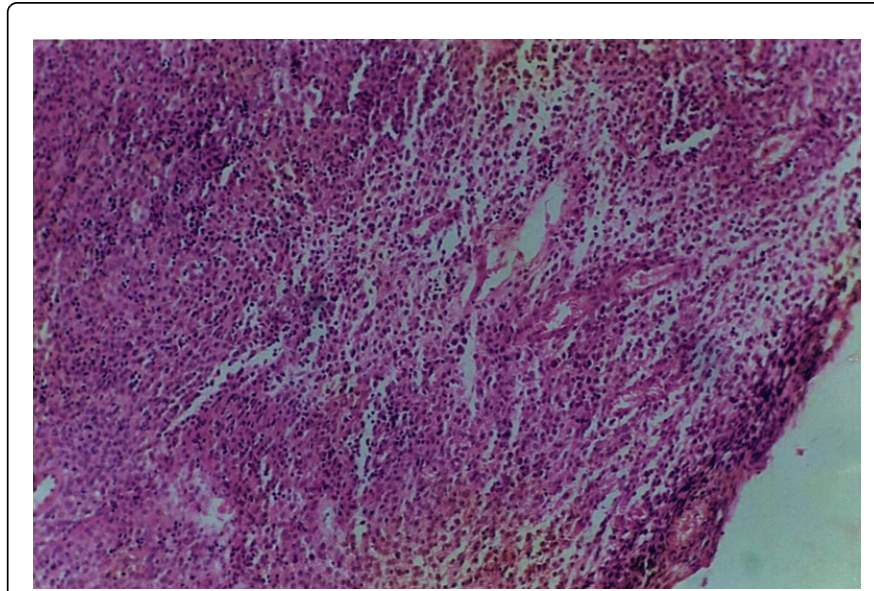

Figure 4: $\mathrm{H}$ and $\mathrm{E}$ section shows numerous histiocyte like cells, eosinophils and lymphocytes in loose connective tissue stroma (low power; 10X).

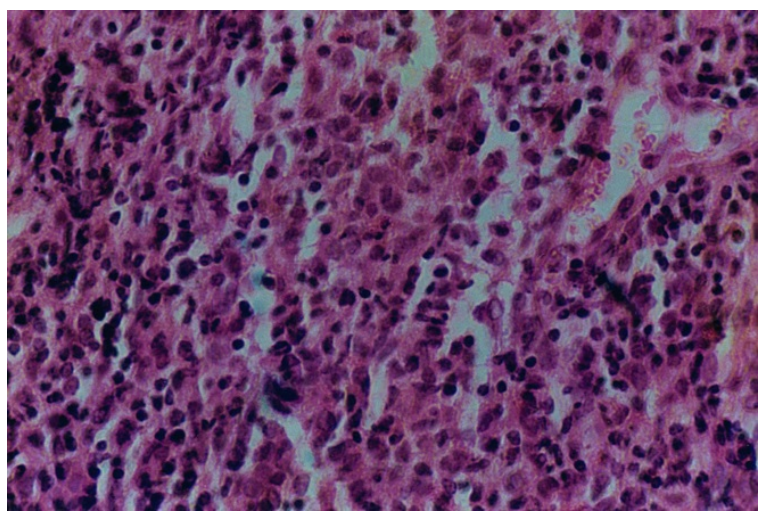

Figure 5: $\mathrm{H}$ and $\mathrm{E}$ section shows numerous histiocyte like cells, eosinophils and lymphocytes in loose connective tissue stroma (high power; $40 \mathrm{X}$ ).

The patient was kept on prednisolone $5 \mathrm{mg}$ q.i.d for three weeks with supportive treatment consisting of antibiotics and eardrops.

On subsequent follow up patient had ear discharge and swelling in head region and was advised bone biopsy. Patients bone biopsy report suggested Histiocytosis $\mathrm{X}$ and patient was advised regular follow up for periodic review. But the patient did not come for subsequent follow up.

\section{Discussion}

Langerhan's cell histiocytosis a chronic disseminated forms i.e. Hand-Schuller-Christian disease is characterized by widespread skeletal and extra skeletal lesions and a chronic clinical course. It occurs usually before the age of five but has been reported even in young adults. It's more common in boys with a gender ratio approximately 2:1. In Hand-Schullers-Christian disease the skeletal system and soft tissues may be involved while in esinophilic granuloma only the bone is affected. Although soft tissue extension is often observed. Letterer-Siwe disease is an acute fulminating disease with 
Citation: Tiwari M (2016) A Rare Case Presentation on Langerhan's Cell Histiocytosis an Chronic Disseminated Form with Gingival Enlargement in Three and a Half Year Old Pediatric Patient. Oral health case Rep 2: 108. doi:10.4172/OHCR.1000108

Page 3 of 3

widespread lesions of both skeletal and extra skeletal tissues including the skin.

Langerhan's cell histiocytosis and chronic disseminated form is characterized by a classic triad of single or multiple punched out bone lesions in the skull, unilateral or bilateral exophthalmos and diabetes insipidus with or without other manifestations like polyuria, dwarfism or infantilism. The complete triad is seen only in $25 \%$ of the affected patients. Involvement of facial bones is frequently associated with soft tissue swelling, tenderness and facial asymmetery. Otitis Media is also common. Other bones frequently involved are femur, ribs, vertebrae, and pelvis. Sometimes the skin exhibits papular or nodular lesions.

Oral manifestations are the earliest signs reported in around 5-75\% of patients. These are often nonspecific and include sore mouth, halitosis, gingivitis, unpleasant taste, loose and sore teeth with their early exfoliation and failure of extracted tooth sockets to heal. Leading to loss of supporting bone mimicking advanced periodontal disease.

Radiographic examination reveals that the individual lesions particularly in the skull are sharply outlined and those in the jaws may be more diffuse exhibiting destruction of alveolar bone with tooth displacement.

Histologically Langerhan's cell histiocytosis a chronic disseminated form (Hand-Schullers-Christian disease) manifests in 4 states during its progression:

- A proliferative histiocytic phase with accumulation of collections of eosinophilic leukocytes scattered throughout the sheets of histiocytosis.

- A vascular granulomatous phase with persistence of histiocytes and eosinophils, sometimes with aggregation of lipid-laden (cholesterol), macrophages.

- A diffuse xanthomatous phase with abundance of "foam cells"

- A fibrous or healing phase [4].
Electron microscopic evaluation of the lesional tissue has been the gold-standard Ultrastructrually; Langerhan's cells contain rod shaped cytoplasmic structures known as birbeck granules which differentiate them from other mononuclear phagocytes. Langerhan's cells show immuno reactivity to CD-1a or CD-207, the latter marker being even more specific to Langerhan's cells. In few cases lesional cells have shown immunoreactivity to S-100 protein and peanut agglutinin (PNA)[5].

Lab investigations often reveal anaemia, leukopenia and thrombocytopenia. The serum cholesterol level is nearly normal. Although tissue cholesterol content may be elevated remarkably.

Treatment of choice is curettage or excision of lesions, inaccessible lesions may be irradiated. Some patients are benefited from chemotherapeutic drugs including prednisolone. One of the significant factors influencing the morbidity and mortality of the disease is the extent of the disease at the time of initial diagnosis and number of organs systems involved [4].

\section{References}

1. Stull M, Kransdorf MJ, Devaney KO (1992) Langerhan's cell histiocytes of Bone from the archives of the AFIP. Radiographics 12: 801-823.

2. Jayachandran S, Murali Gopika Mohan GV (1999) Langerhans cell disease-A case report. JIAOMR 10(1): 9-13.

3. Martinez-Pereda CM, Rodriguez VG, Moya BG, Garcia CM (2009) Langerhan's cell histiocytosis: Literature review and descriptive analysis of oral manifestations. Med Oral Pathol Oral Cir Buccal 14: 222-228.

4. Rajendran R, Sivapathasundaram B (2009) Hand Schuller Christian Disease: Diseases of Specific Systems. (6thedn), Elsevier; Shafer's Textbook of Oral pathology 744-745.

5. Neville BW, Damm DD, Allen CM, Bouquot JE (2009) Langerhans cell Histiocytosis (3rdedn), Elsevier, Textbook of Oral and Maxillofacial Pathology 590-592. 